\title{
$b$-Metric Generalization of Some Fixed Point Theorems
}

\author{
Mumtaz Ali (iD) and Muhammad Arshad (DD \\ Department of Mathematics, International Islamic University, H-10, Islamabad 44000, Pakistan \\ Correspondence should be addressed to Mumtaz Ali; mumtaz6767@yahoo.com
}

Received 27 December 2017; Revised 7 March 2018; Accepted 11 March 2018; Published 15 April 2018

Academic Editor: Pasquale Vetro

Copyright (C) 2018 Mumtaz Ali and Muhammad Arshad. This is an open access article distributed under the Creative Commons Attribution License, which permits unrestricted use, distribution, and reproduction in any medium, provided the original work is properly cited.

We prove common fixed point theorems in terms of $b$-metric spaces with new contractions. The results presented in this paper include $b$-metric generalizations of some fixed point theorems of Fisher, Pachpatte, and Sahu and Sharma.

\section{Introduction}

Fixed point theory is a fascinating topic for research in both analysis and topology. In this direction the Banach contraction mapping theorem of 1922 popularly known as Banach contraction mapping principle is a rewarding result in fixed point theory. It has widespread applications in both pure and applied mathematics. The well-known Banach [1] contraction mapping principle states that if " $X$ is a complete metric space and $T: X \rightarrow X$ is a contraction mapping of $X$ into itself then $T$ has unique fixed point in $X$." This celebrated principle has been generalized by several authors. In 1989, Bakhtin [2] introduced the concept of $b$-metric space which is generalization of renowned Banach contraction mapping principle. Czerwik $[3,4]$ extended the concept of $b$-metric space in 1993. Bakhtin's concept of $b$-metric spaces has been extensively generalized and improved by several mathematicians for fixed points in several different ways, namely, Boriceanu [5], Bota et al. [6], Chen et al. [7], Hussain and Shah [8], Kutbi et al. [9], and Shukla [10] to name a few. In this paper, our main concern is to study common fixed point theorems in complete $b$-metric spaces for three self-mappings. The obtained results are generalizations of $b$ metric variant of fixed point theorems of Fisher, Pachpatte, and Sahu and Sharma.

The following fixed point theorems were proved in [1113].
Theorem 1 (see [11]). Let $T$ be a mapping of the complete metric space $X$ into itself satisfying the inequality

$$
\begin{aligned}
(d(T x, T y))^{2} \leq & \alpha_{1}(d(x, T x) d(y, T y)) \\
& +\alpha_{2}(d(x, T y) d(y, T x)),
\end{aligned}
$$

$\forall x, y \in X, 0 \leq \alpha_{1}<1,0 \leq \alpha_{2}$ then $T$ has a fixed point in $X$.

Theorem 2 (see [12]). If $T$ is a mapping of the complete metric space $X$ into itself satisfying the inequality

$$
\begin{aligned}
& {[d(T x, T y)]^{2}} \\
& \quad \leq \alpha_{1}[d(x, T x) d(y, T y)+d(x, T y) d(y, T x)] \\
& \quad+\alpha_{2}[d(x, T x) d(y, T x)+d(x, T y) d(y, T y)],
\end{aligned}
$$

for all $x, y \in X$, where $\alpha_{1}, \alpha_{2} \geq 0$ and $\alpha_{1}+2 \alpha_{2}<1$ then $T$ has a unique fixed point.

Theorem 3 (see [13]). If T is a mapping of the complete metric space $X$ into itself satisfying the inequality

$$
\begin{aligned}
& {[d(T x, T y)]^{2}} \\
& \leq \alpha_{1}[d(x, T x) d(y, T y)+d(x, T y) d(y, T x)] \\
& \quad+\alpha_{2}[d(x, T x) d(y, T x)+d(x, T y) d(y, T y)] \\
& \quad+\alpha_{3}\left[\{d(y, T x)\}^{2}+\{d(y, T y)\}^{2}\right],
\end{aligned}
$$


for all $x, y$ in $X$, where $\alpha_{1}, \alpha_{2}, \alpha_{3} \geq 0$ and $\alpha_{1}+2 \alpha_{2}+\alpha_{3}<1$, then $T$ has a unique fixed point.

\section{Preliminaries}

In this section we recall some basic definitions and necessary results from existing literature that will be used in the sequel.

Definition 4 (see [3]). Let $X$ be a nonempty set and $s \geq 1$ be a given real number. A function $d: X \times X \rightarrow R^{+}$is said to be a $b$-metric on $X$ if the following conditions hold:

(i) $d(x, y)=0$ if and only if $x=y$.

(ii) $d(x, y)=d(y, x)$ for all $x, y \in X$.

(iii) $d(x, y) \leq s(d(x, z)+d(z, y))$ for all $x, y, z \in X$.

The pair $(X, d)$ is called $b$-metric space.

It is clear from the definition of $b$-metric that every metric space is $b$-metric for $s=1$, but the converse need not be true. The following example illustrates the above remarks.

Example 5 (see [5]). Let $X=\{0,1,2\}$. Define $d: X \times X \rightarrow$ $R^{+}$by $d(0,0)=d(1,1)=d(2,2)=0, d(1,2)=d(2,1)=$ $d(1,0)=d(0,1)=1$, and $d(0,2)=d(2,0)=m \geq 2$ for $s=m / 2$. The function defined above is a $b$-metric space but is not a metric space for $m>2$.

Proposition 6 (see [14]). Let $X$ be a nonempty set and the mappings $f, g, h: X \rightarrow X$ have a unique point of coincidence in $X$. If $\{f, h\}$ and $\{g, h\}$ are weakly compatible self-maps of $X$, then $f, g, h$ have a unique common fixed point.

Definition 7. A sequence $\left\{x_{n}\right\}$ in a $b$-metric space $(X, d)$ is called Cauchy sequence if and only if $\lim _{m, n \rightarrow \infty} d\left(x_{n}, x_{m}\right)=0$.

Definition 8. A sequence $\left\{x_{n}\right\}$ in a $b$-metric space $(X, d)$ is said to converge to a point $x \in X$ if and only if $\lim _{n \rightarrow \infty} d\left(x_{n}, x\right)=0$. We denote this by $\lim _{n \rightarrow \infty} x_{n}=x$.

Definition 9. A $b$-metric space $(X, d)$ is said to be complete if and only if every Cauchy sequence in $X$ converges to a point of $X$.

Definition 10 (see [15]). Let $f$ and $g$ be self-mappings of a set $X$. If $z=f x=g x$ for some $x$ in $X$, then $x$ is called a coincidence point of $f$ and $g$ and $z$ is called a point of coincidence of $f$ and $g$.

Definition 11 (see [16]). The mappings $f, g: X \rightarrow X$ are weakly compatible, if, for every $x \in X$, the following holds: $f(g x)=g(f x)$ whenever $f x=g x$.

Definition 12. A point $x \in X$ is said to be a fixed point of a self-map $T: X \rightarrow X$ if $T(x)=x$.

\section{Main Results}

In this section we obtain coincidence points and common fixed point theorems for three maps in complete $b$-metric spaces. In order to start our main results we begin with a simple but useful Lemma.
Lemma 13. Let $(X, d)$ be a complete $b$-metric space with the coefficient $s \geq 1$ and let $f, g, h: X \rightarrow X$ be self-mappings from $X$ into itself satisfying the following conditions:

(i) $f(X) \cup g(X) \subseteq h(X)$.

(ii)

$$
\begin{aligned}
& {[d(f x, g y)]^{2} \leq \alpha_{1}[d(h x, f x) d(h y, g y)} \\
& \quad+d(h x, g y) d(h y, f x)]+\alpha_{2}[d(h y, g x) d(h y, f x) \\
& \quad+d(h x, g y) d(h y, g y)]+\alpha_{3}\left[\{d(h y, f x)\}^{2}\right. \\
& \left.\quad+\{d(h y, g y)\}^{2}\right]
\end{aligned}
$$

for all $x, y \in X ; \alpha_{1}, \alpha_{2}, \alpha_{3} \geq 0$, such that

$$
s \alpha_{1}+\left(s^{2}+s\right) \alpha_{2}+\alpha_{3}<1
$$

Then every sequence $\left\{y_{n}\right\}$ with initial point $x_{0}$ is a Cauchy sequence in $X$.

Proof. Let $x_{0} \in X$ and choose a point $x_{1} \in X$ such that $h x_{1}=$ $f x_{0}$ and for $x_{1}$ there exists $x_{2} \in X$ such that $h x_{2}=g x_{1}$, and continuing this process we construct sequences $\left\{x_{n}\right\}$ and $\left\{y_{n}\right\}$ in $X$ given by

$$
\begin{aligned}
y_{2 n} & =h x_{2 n+1}=f x_{2 n}, \\
y_{2 n+1} & =h x_{2 n+1}=g x_{2 n+1} \quad \forall n \geq 0 .
\end{aligned}
$$

Suppose that there exists $h \in[0,1 / s)$ such that

$$
d\left(y_{n}, y_{n+1}\right) \leq h d\left(y_{n-1}, y_{n}\right) \quad \forall n \geq 1
$$

We show that $\left\{y_{n}\right\}$ is a Cauchy sequence in $X$. Using (4), we have

$$
\begin{aligned}
& {\left[d\left(y_{2 n}, y_{2 n+1}\right)\right]^{2}=\left[d\left(h x_{2 n+1}, h x_{2 n+2}\right)\right]^{2}} \\
& \quad=\left[d\left(f x_{2 n}, g x_{2 n+1}\right)\right]^{2} \\
& \quad \leq \alpha_{1}\left[d\left(h x_{2 n}, f x_{2 n}\right) d\left(h x_{2 n+1}, g x_{2 n+1}\right)\right. \\
& \left.\quad+d\left(h x_{2 n}, g x_{2 n+1}\right) d\left(h x_{2 n+1}, f x_{2 n}\right)\right] \\
& \quad+\alpha_{2}\left[d\left(h x_{2 n+1}, g x_{2 n}\right) d\left(h x_{2 n+1}, f x_{2 n}\right)\right. \\
& \left.\quad+d\left(h x_{2 n}, g x_{2 n+1}\right) d\left(h x_{2 n+1}, g x_{2 n+1}\right)\right] \\
& \quad+\alpha_{3}\left[\left\{d\left(h x_{2 n+1}, f x_{2 n}\right)\right\}^{2}+\left\{d\left(h x_{2 n+1}, g x_{2 n+1}\right)\right\}^{2}\right] \\
& \quad \leq \alpha_{1}\left[d\left(h x_{2 n}, h x_{2 n+1}\right) d\left(h x_{2 n+1}, h x_{2 n+2}\right)\right. \\
& \left.\quad+d\left(h x_{2 n}, h x_{2 n+2}\right)\left(d\left(h x_{2 n+1}, h x_{2 n+1}\right)\right)\right] \\
& \quad+\alpha_{2}\left[d\left(h x_{2 n}, h x_{2 n+!}\right) d\left(h x_{2 n+1}, h x_{2 n+1}\right)\right. \\
& \left.\quad+d\left(h x_{2 n} h x_{2 n+2}\right) d\left(h x_{2 n+1}, h x_{2 n+2}\right)\right] \\
& \quad+\alpha_{3}\left[\left\{d\left(h x_{2 n+1}, h x_{2 n+1}\right)\right\}^{2}+\left\{d\left(h x_{2 n+1}, h x_{2 n+2}\right)\right\}^{2}\right]
\end{aligned}
$$




$$
\begin{aligned}
& \leq \alpha_{1}\left[d\left(y_{2 n-1}, y_{2 n}\right) d\left(y_{2 n}, y_{2 n+1}\right)\right. \\
& \left.+d\left(y_{2 n-1}, y_{2 n+1}\right) d\left(y_{2 n}, y_{2 n}\right)\right] \\
& +\alpha_{2}\left[d\left(y_{2 n-1}, y_{2 n}\right) d\left(y_{2 n}, y_{2 n}\right)\right. \\
& \left.+d\left(y_{2 n-1}, y_{2 n+1}\right) d\left(y_{2 n}, y_{2 n+1}\right)\right] \\
& +\alpha_{3}\left[\left\{d\left(y_{2 n}, y_{2 n}\right)\right\}^{2}+\left\{d\left(y_{2 n}, y_{2 n+1}\right)\right\}^{2}\right] \\
& \leq \alpha_{1} d\left(y_{2 n-1}, y_{2 n}\right)+\alpha_{2} d\left(y_{2 n-1}, y_{2 n+1}\right) \\
& +\alpha_{3} d\left(y_{2 n}, y_{2 n+1}\right) \leq \alpha_{1} d\left(y_{2 n-1}, y_{2 n}\right) \\
& +s \alpha_{2}\left[d\left(y_{2 n-1}, y_{2 n}\right)+d\left(y_{2 n}, y_{2 n+1}\right)\right] \\
& +\alpha_{3} d\left(y_{2 n}, y_{2 n+1}\right) \leq\left(\alpha_{1}+s \alpha_{2}\right) d\left(y_{2 n-1}, y_{2 n}\right) \\
& +\left(s \alpha_{2}+\alpha_{3}\right) d\left(y_{2 n}, y_{2 n+1}\right) \\
{[1} & \left.-\left(s \alpha_{2}+\alpha_{3}\right)\right] d\left(y_{2 n}, y_{2 n+1}\right) \leq\left(\alpha_{1}\right. \\
& \left.+s \alpha_{2}\right) d\left(y_{2 n-1}, y_{2 n}\right) \Longrightarrow \\
d( & \left.y_{2 n}, y_{2 n+1}\right) \leq \frac{\left(\alpha_{1}+s \alpha_{2}\right)}{\left[1-\left(s \alpha_{2}+\alpha_{3}\right)\right]} d\left(y_{2 n-1}, y_{2 n}\right) \\
& \leq \lambda d\left(y_{2 n-1}, y_{2 n}\right),
\end{aligned}
$$

where

$$
\begin{aligned}
\lambda & =\frac{\left(\alpha_{1}+s \alpha_{2}\right)}{\left[1-\left(s \alpha_{2}+\alpha_{3}\right)\right]}<1, \\
d\left(y_{2 n}, y_{2 n+1}\right) & \leq \lambda d\left(y_{2 n}, y_{2 n-1}\right), \\
d\left(y_{2 n}, y_{2 n+1}\right) & \leq \lambda^{2} d\left(y_{2 n-1}, y_{2 n-2}\right) .
\end{aligned}
$$

Therefore, for all $n \in \mathbb{N}$, we can write

$$
d\left(y_{n+1}, y_{n+2}\right) \leq \lambda d\left(y_{n}, y_{n+1}\right) \leq \cdots \leq \lambda^{n+1} d\left(y_{0}, y_{1}\right) .
$$

Now, for any $m, n \in \mathbb{N}, m>n$, we have

$$
\begin{aligned}
& d\left(y_{n}, y_{m}\right) \leq s d\left(y_{n}, y_{n+1}\right)+s d\left(y_{n+1}, y_{m}\right) \\
& \leq s d\left(y_{n}, y_{n+1}\right)+s^{2} d\left(y_{n+1}, y_{n+2}\right)+s^{3} d\left(y_{n+2}, y_{m}\right) \\
& \leq s d\left(y_{n}, y_{n+1}\right)+s^{2} d\left(y_{n+1}, y_{n+2}\right)+s^{3} d\left(y_{n+2}, y_{n+3}\right) \\
&+\cdots+s^{m-n-1} d\left(y_{m-2}, y_{m-1}\right)+s^{m-n-1} d\left(y_{m-1}, y_{m}\right) \\
& \leq s d\left(y_{n}, y_{n+1}\right)+s^{2} d\left(y_{n+1}, y_{n+2}\right)+s^{3} d\left(y_{n+2}, y_{n+3}\right) \\
&+\cdots+s^{m-n-1} d\left(y_{m-2}, y_{m-1}\right)+s^{m-n} d\left(y_{m-1}, y_{m}\right) \\
& \leq {\left[s \lambda^{n}+s^{2} \lambda^{n+1}+s^{3} \lambda^{n+2}+\cdots+s^{m-n} \lambda^{m-1}\right] d\left(y_{1}, y_{0}\right) } \\
& \leq \frac{s \lambda^{n}}{(1-s \lambda)} d\left(y_{1}, y_{0}\right) .
\end{aligned}
$$

Therefore, we have

$$
d\left(y_{n}, y_{m}\right) \leq \frac{s \lambda^{n}}{(1-s \lambda)} d\left(y_{0}, y_{1}\right) \longrightarrow 0 \quad \text { as } n \longrightarrow \infty
$$

Thus,

$$
d\left(y_{n}, y_{m}\right) \longrightarrow 0 \quad \text { as } n \longrightarrow \infty
$$

Hence $\left\{y_{n}\right\}$ is a Cauchy sequence in $b$-metric space $X$. [13].

The next theorem is $b$-metric variant of Theorem 1.3 in

Theorem 14. Let $(X, d)$ be a complete $b$-metric space with the coefficient $s \geq 1$. Suppose the self-maps $f, g, h: X \rightarrow X$ satisfy the condition

$$
\begin{aligned}
& {[d(f x, g y)]^{2} \leq \alpha_{1}[d(h x, f x) d(h y, g y)} \\
& \quad+d(h x, g y) d(h y, f x)] \\
& \quad+\alpha_{2}[d(h y, g x) d(h y, f x) \\
& \quad+d(h x, g y) d(h y, g y)]+\alpha_{3}\left[\{d(h y, f x)\}^{2}\right. \\
& \left.\quad+\{d(h y, g y)\}^{2}\right],
\end{aligned}
$$

where $\alpha_{1}, \alpha_{2}, \alpha_{3} \geq 0$ are nonnegative reals with

$$
s \alpha_{1}+\left(s^{2}+s\right) \alpha_{2}+\alpha_{3}<1
$$

If $f(X) \cup g(X) \subseteq h(X)$ and $h(X)$ is a complete subspace of $X$, then the maps $f, g$, and $h$ have a coincidence point $u$ in $X$. Moreover, if $\{f, h\}$ and $\{g, h\}$ are weakly compatible pairs. Then $f, g$, and h have a unique common fixed point in $X$.

Proof. Let $x_{0}$ be an arbitrary point in $X$ and define the sequence $\left\{y_{n}\right\}$ in $X$ such that $y_{2 n}=h x_{2 n+1}=f x_{2 n}$ and $y_{2 n+1}=h x_{2 n+2}=g x_{2 n+1} \forall n=0,1,2, \ldots$. Now, we show that $\left\{y_{n}\right\}$ is a Cauchy sequence. So, by (4), we have

$$
\begin{aligned}
& {\left[d\left(y_{2 n}, y_{2 n+1}\right)\right]^{2}=\left[d\left(f x_{2 n}, g x_{2 n+1}\right)\right]^{2}} \\
& \quad \leq \alpha_{1}\left[d\left(h x_{2 n}, f x_{2 n}\right) d\left(h x_{2 n+1}, g x_{2 n+1}\right)\right. \\
& \left.\quad+d\left(h x_{2 n}, g x_{2 n+1}\right) d\left(h x_{2 n+1}, f x_{2 n}\right)\right] \\
& \quad+\alpha_{2}\left[d\left(h x_{2 n+1}, g x_{2 n}\right) d\left(h x_{2 n+1}, f x_{2 n}\right)\right. \\
& \left.\quad+d\left(h x_{2 n}, g x_{2 n+1}\right) d\left(h x_{2 n+1}, g x_{2 n+1}\right)\right] \\
& \quad+\alpha_{3}\left[\left\{d\left(h x_{2 n+1}, f x_{2 n}\right)\right\}^{2}+\left\{d\left(h x_{2 n+1}, g x_{2 n+1}\right)\right\}^{2}\right] \\
& \quad \leq \alpha_{1}\left[d\left(y_{2 n-1}, y_{2 n}\right) d\left(y_{2 n}, y_{2 n+1}\right)\right. \\
& \left.\quad+d\left(y_{2 n-1}, y_{2 n}\right) d\left(y_{2 n}, y_{2 n}\right)\right] \\
& \quad+\alpha_{2}\left[d\left(y_{2 n}, y_{2 n}\right) d\left(y_{2 n}, y_{2 n}\right)\right. \\
& \left.\quad+d\left(y_{2 n-1}, y_{2 n+1}\right) d\left(y_{2 n}, y_{2 n+1}\right)\right] \\
& \quad+\alpha_{3}\left[\left\{d\left(y_{2 n}, y_{2 n}\right)\right\}^{2}+\left\{d\left(y_{2 n}, y_{2 n+1}\right)\right\}^{2}\right]
\end{aligned}
$$




$$
\begin{aligned}
& \quad \alpha_{1}\left[d\left(y_{2 n-1}, y_{2 n}\right) d\left(y_{2 n}, y_{2 n+1}\right)\right] \\
& \quad+\alpha_{2}\left[d\left(y_{2 n-1}, y_{2 n+1}\right) d\left(y_{2 n}, y_{2 n+1}\right)\right] \\
& \quad+\alpha_{3}\left\{d\left(y_{2 n}, y_{2 n+1}\right)\right\}^{2} \Longrightarrow \\
& d\left(y_{2 n}, y_{2 n+1}\right) \leq \alpha_{1} d\left(y_{2 n-1}, y_{2 n}\right)+\alpha_{2} d\left(y_{2 n-1}, y_{2 n+1}\right) \\
& \quad+\alpha_{3} d\left(y_{2 n}, y_{2 n+1}\right) \leq \alpha_{1} d\left(y_{2 n-1}, y_{2 n}\right) \\
& \quad+s \alpha_{2}\left[d\left(y_{2 n-1}, y_{2 n}\right)+d\left(y_{2 n}, y_{2 n+1}\right)\right] \\
& \quad+\alpha_{3} d\left(y_{2 n}, y_{2 n+1}\right) \leq\left(\alpha_{1}+s \alpha_{2}\right) d\left(y_{2 n}, y_{2 n+1}\right) \\
& \quad+\left(s \alpha_{2}+\alpha_{3}\right) d\left(y_{2 n-1}, y_{2 n}\right) \Longrightarrow \\
& d\left(y_{2 n}, y_{2 n+1}\right) \leq \frac{\left(\alpha_{1}+s \alpha_{3}\right)}{\left[1-\left(s \alpha_{2}+\alpha_{3}\right)\right]} d\left(y_{2 n-1}, y_{2 n}\right) \\
& \quad \leq \lambda d\left(y_{2 n-1}, y_{2 n}\right) \Longrightarrow \\
& d\left(y_{2 n}, y_{2 n+1}\right) \leq \lambda d\left(y_{2 n-1}, y_{2 n}\right)
\end{aligned}
$$

Similarly, we can show that

$$
d\left(y_{2 n+1}, y_{2 n+2}\right) \leq \lambda^{2} d\left(y_{2 n}, y_{2 n+1}\right),
$$

where

$$
\lambda=\frac{\left(\alpha_{1}+s \alpha_{2}\right)}{\left[1-\left(s \alpha_{2}+\alpha_{3}\right)\right]}<\frac{1}{s} .
$$

Therefore, for all $m, n \in \mathbb{N}$ with $m>n$, we can get

$$
\begin{aligned}
\left(y_{n+1}, y_{n+2}\right) \leq & \lambda d\left(y_{n}, y_{n+1}\right) \leq \cdots \leq \lambda^{n+1} d\left(y_{0}, y_{1}\right) \\
d\left(y_{n}, y_{m}\right) \leq & s d\left(y_{n}, y_{n+1}\right)+s d\left(y_{n+1}, y_{m}\right) \\
\leq & s d\left(y_{n}, y_{n+1}\right)+s^{2} d\left(y_{n+1}, y_{n+2}\right) \\
& +s^{3} d\left(y_{n+2}, y_{m}\right) \\
\leq & s d\left(y_{n}, y_{n+1}\right)+s^{2} d\left(y_{n+1}, y_{n+2}\right) \\
& +s^{3} d\left(y_{n+2}, y_{n+3}\right)+s^{4} d\left(y_{n+3}, y_{m}\right) \\
\leq & s d\left(y_{n}, y_{n+1}\right)+s^{2} d\left(y_{n+1}, y_{n+2}\right) \\
& +s^{3} d\left(y_{n+2}, y_{n+3}\right)+\cdots \\
& +s^{m-n-2} d\left(y_{m-3}, y_{m-2}\right) \\
& +s^{m-n-1} d\left(y_{m-2}, y_{m-1}\right) \\
& +s^{m-n} d\left(y_{m-1}, y_{m}\right) \Longrightarrow \\
d\left(y_{n}, y_{m}\right) \leq & \frac{s \lambda^{n}}{(1-s \lambda)} d\left(y_{0}, y_{1}\right) .
\end{aligned}
$$

Thus, as $n \rightarrow \infty, d\left(y_{n}, y_{m}\right) \rightarrow 0$. It follows from Lemma 13 that $\left\{y_{n}\right\}$ is a Cauchy sequence and, by the completeness of $X$, $\left\{y_{n}\right\}$ converges to some $y \in X$. Therefore,

$$
\begin{aligned}
\lim _{n \rightarrow \infty} f x_{2 n} & =\lim _{n \rightarrow \infty} g x_{2 n+1}=\lim _{n \rightarrow \infty} h x_{2 n+1}=\lim _{n \rightarrow \infty} h x_{2 n+2} \\
& =y .
\end{aligned}
$$

Thus, $f(X) \cup g(X) \subseteq h(X)$ implies either $f(X) \subseteq h(X)$ or $g(X) \subseteq h(X)$.

Case 1. Let $f(X) \subseteq h(X)$. Since $h(X)$ is a complete subspace of $f(X) \cup g(X)$ and $f(X) \subseteq h(X)$ implies $h(X)$ is closed, hence, there exist $v, y \in X$ such that $h v=y$. If $g v \neq y$, then, by using (14), we get

$$
\begin{aligned}
& {\left[d\left(f x_{2 n}, g v\right)\right]^{2} \leq \alpha_{1}\left[d\left(h x_{2 n}, f x_{2 n}\right) d(h v, g v)\right.} \\
& \left.\quad+d\left(h x_{2 n}, g v\right) d\left(h v, f x_{2 n}\right)\right] \\
& +\alpha_{2}\left[d\left(h v, g x_{2 n}\right) d\left(h v, f x_{2 n}\right)\right. \\
& \left.\quad+d\left(h x_{2 n}, g v\right) d(h v, g v)\right]+\alpha_{3}\left[\left\{d\left(h v, f x_{2 n}\right)\right\}^{2}\right. \\
& \left.\quad+\{d(h v, g v)\}^{2}\right] .
\end{aligned}
$$

Taking limit as $n \rightarrow \infty$ yields

$$
\begin{aligned}
& {\left[d\left(f x_{2 n}, g v\right)\right]^{2} \leq \alpha_{1}\left[d\left(h x_{2 n}, f x_{2 n}\right) d(h v, g v)\right.} \\
& \left.\quad+d\left(h x_{2 n}, g v\right) d\left(h v, f x_{2 n}\right)\right] \\
& +\alpha_{2}\left[d\left(h v, g x_{2 n}\right) d\left(h v, f x_{2 n}\right)\right. \\
& \left.\quad+d\left(h x_{2 n}, g v\right) d(h v, g v)\right]+\alpha_{3}\left[\left\{d\left(h v, f x_{2 n}\right)\right\}^{2}\right. \\
& \left.\quad+\{d(h v, g v)\}^{2}\right] \\
& {[d(y, g v)]^{2} \leq \alpha_{1}[d(y, y) d(y, g v)} \\
& \quad+d(y, g v) d(y, y)] \\
& +\alpha_{2}[d(y, y) d(y, y)+d(y, g v) d(y, g v)] \\
& \quad+\alpha_{3}\left[\{d(y, y)\}^{2}+\{d(y, g v)\}^{2}\right] \leq\left(\alpha_{2}+\alpha_{3}\right) \\
& \quad+[d(y, g v)]^{2} \Longrightarrow \\
& {\left[1-\left(\alpha_{2}+\alpha_{3}\right)\right][d(y, g v)]^{2} \leq 0,}
\end{aligned}
$$

and the above inequality is possible only if $[d(y, g y)]^{2}=0$ which implies that $y=g v$. It follows that

$$
h v=y=g v .
$$

Since $g$ and $h$ are weakly compatible, we have $h g v=g h v$ and so

$$
g y=h y .
$$


If $y \neq g y$, then by (14) we have

$$
\begin{aligned}
& {[d(}\left.\left.f x_{2 n}, g y\right)\right]^{2} \\
& \leq \alpha_{1} {\left[d\left(h x_{2 n}, f x_{2 n}\right) d(h y, g y)+d\left(h x_{2 n}, g y\right) d\left(h y, f x_{2 n}\right)\right] } \\
&+ \alpha_{2}\left[d\left(h y, g x_{2 n}\right) d\left(h y, f x_{2 n}\right)+d\left(h x_{2 n}, g y\right) d(h y, g y)\right] \\
&+ \alpha_{3}\left[\left\{d\left(h y, f x_{2 n}\right)\right\}^{2}+\{d(h y, g y)\}^{2}\right] . \\
& \text { As } n \rightarrow \infty, \text { we have } \\
& {[d(y, g y)]^{2} } \\
& \leq \alpha_{1}[d(y, y) d(h y, g y)+d(y, g y) d(h y, y)] \\
& \quad+\alpha_{2}[d(h y, y) d(h y, y)+d(y, g y) d(h y, g y)] \\
& \quad+\alpha_{3}\left[\{d(h y, y)\}^{2}+\{d(h y, g y)\}^{2}\right] \\
& \leq \alpha_{1}[d(y, y) d(g y, g y)+d(y, g y) d(g y, y)] \\
& \quad+\alpha_{2}[d(g y, y) d(g y, y)+d(y, g y) d(g y, g y)] \\
& \quad+\alpha_{3}\left[\{d(g y, y)\}^{2}+\{d(g y, g y)\}^{2}\right] \\
& \leq\left(\alpha_{1}+\alpha_{2}+\alpha_{3}\right)[d(y, g y)]^{2} \Longrightarrow[d(y, g y)]^{2} \\
& {\left[1-\left(\alpha_{1}+\alpha_{2}+\alpha_{3}\right)[d(y, g y)]^{2} \Longrightarrow\right.} \\
&\left.\left.\quad+\alpha_{2}+\alpha_{3}\right)\right][d(y, g y)]^{2} \leq 0,
\end{aligned}
$$

and the inequality is possible only if

$$
\begin{aligned}
d(y, g y) & =0 \Longrightarrow \\
y & =g y .
\end{aligned}
$$

By using (24), we have

$$
h y=g y=y .
$$

Case 2. If $g(X) \subseteq h(X)$, again there exists points $v, y \in X$ such that $y=h v$ if $y \neq f v$, and then by using (14) we get

$$
\begin{aligned}
& {[d(f v, g y)]^{2} \leq \alpha_{1}[d(h v, f v) d(h y, g y)} \\
& \quad+d(h v, g y) d(h y, f v)] \\
& \quad+\alpha_{2}[d(h y, g v) d(h y, f v) \\
& \quad+d(h v, g y) d(h y, g y)]+\alpha_{3}\left[\{d(h y, f v)\}^{2}\right. \\
& \left.\quad+\{d(h y, g y)\}^{2}\right] .
\end{aligned}
$$

It follows that

$$
\begin{aligned}
& {[d(f v, y)]^{2}} \\
& \quad \leq \alpha_{1}[d(y, f v) d(y, y)+d(y, y) d(y, f v)] \\
& \quad+\alpha_{2}[d(y, y) d(y, f v)+d(y, y) d(y, y)] \\
& \quad+\alpha_{3}\left[\{d(y, f v)\}^{2}+\{d(y, y)\}^{2}\right] \Longrightarrow \\
& \quad\left(1-\alpha_{3}\right)[d(y, f v)]^{2} \leq 0,
\end{aligned}
$$

which is possible only if $d(y, f y)=0 \Rightarrow y=f v$ :

$$
\Longrightarrow f v=h v=y \text {. }
$$

Since $f$ and $h$ are weakly compatible and hence $f h v=h f v$ and so $f y=h y$ and by (28), we have

$$
y=f y=g y=h y .
$$

Thus, $y$ is the common fixed point of self-mappings $f, g$, and $h$. This completes the proof of the theorem.

Uniqueness. In order to prove uniqueness, let $y_{1} \neq y_{2}$ be two distinct common fixed points of the self-maps $f, g$, and $h$ then we have by (14)

$$
\begin{aligned}
& {\left[d\left(y_{1}, y_{2}\right)\right]^{2}=\left[d\left(f y_{1}, g y_{2}\right)\right]^{2}} \\
& \quad \leq \alpha_{1}\left[d\left(h y_{1}, f y_{1}\right) d\left(h y_{2}, g y_{2}\right)\right. \\
& \left.\quad+d\left(h y_{1}, g y_{2}\right) d\left(h y_{2}, f y_{1}\right)\right] \\
& \quad+\alpha_{2}\left[d\left(h y_{2}, g y_{1}\right) d\left(h y_{2}, f y_{1}\right)\right. \\
& \left.\quad+d\left(h y_{1}, g y_{2}\right) d\left(h y_{2}, g y_{2}\right)\right]+\alpha_{3}\left[\left\{d\left(h y_{2}, f y_{1}\right)\right\}^{2}\right. \\
& \left.\quad+\left\{d\left(h y_{2}, g y_{2}\right)\right\}^{2}\right] \leq \alpha_{1}\left[d\left(y_{1}, y_{1}\right) d\left(y_{2}, y_{2}\right)\right. \\
& \left.\quad+d\left(y_{1}, y_{2}\right) d\left(y_{1}, y_{2}\right)\right]+\alpha_{2}\left[d\left(y_{2}, y_{1}\right) d\left(y_{2}, y_{1}\right)\right. \\
& \left.\quad+d\left(y_{1}, y_{2}\right) d\left(y_{2}, y_{2}\right)\right]+\alpha_{3}\left[\left\{d\left(y_{1}, y_{2}\right)\right\}^{2}\right. \\
& \left.\quad+\left\{d\left(y_{2}, y_{2}\right)\right\}^{2}\right] \leq\left(\alpha_{1}+\alpha_{2}+\alpha_{3}\right)\left[d\left(y_{1}, y_{2}\right)\right]^{2} \Longrightarrow \\
& {\left[1-\left(\alpha_{1}+\alpha_{2}+\alpha_{3}\right)\right]\left[d\left(y_{1}, y_{2}\right)\right]^{2} \leq 0,}
\end{aligned}
$$

which is possible only if $d\left(y_{1}, y_{2}\right)=0 \Rightarrow y_{1}=y_{2}$ which gives us uniqueness of $y$.

Remark 15. If we put $\alpha_{3}=0$, then we get Theorem 1.2 in [12].

Now we present the modified form of Theorem 3 in terms of $b$-metric spaces.

Theorem 16. Let $(X, d)$ be a complete $b$-metric space with the coefficient $s \geq 1$ and suppose the self-maps $f, g, h: X \rightarrow X$ satisfy the condition

$$
\begin{aligned}
& {[d(f x, g y)]^{2}} \\
& \leq \alpha_{1}[d(h x, f x) d(h y, g y)] \\
& \quad+\alpha_{2}[d(h x, g y) d(h y, g y)(1+d(f x, h y))] \\
& \quad+\alpha_{3}[d(h x, h y) d(h y, g y)(1+d(f x, h y))] \\
& \quad+\alpha_{4}[d(h x, g y) d(h y, g y)] \\
& \quad+\alpha_{5}\left[\{d(h y, f x)\}^{2}+\{d(h y, g y)\}^{2}\right],
\end{aligned}
$$

where $\alpha_{1}, \alpha_{2}, \alpha_{3}, \alpha_{4}, \alpha_{5} \geq 0$ are nonnegative reals with

$$
s \alpha_{1}+\left(s^{2}+s\right) \alpha_{2}+s \alpha_{3}+\left(s^{2}+s\right) \alpha_{4}+\alpha_{5}<1 .
$$


If $f(X) \cup g(X) \subseteq h(X)$ and $h(X)$ is a complete subspace of $X$, then the maps $f, g$, and $h$ have a coincidence point $u$ in $X$. Moreover, if $\{f, h\}$ and $\{g, h\}$ are weakly compatible, then $f, g$, and $h$ have a unique common fixed point in $X$.

Proof. Let $x_{0} \in X$ and define a sequence of points $\left\{y_{n}\right\}$ in $X$ as follows, $y_{2 n}=h x_{2 n+1}=f x_{2 n}$ and $y_{2 n+1}=h x_{2 n+2}=$ $g x_{2 n+1} \forall n=0,1,2, \ldots$. By using (34), we have

$$
\begin{aligned}
& {\left[d\left(y_{2 n}, y_{2 n+1}\right)\right]^{2}=\left[d\left(f x_{2 n}, g x_{2 n+1}\right)\right]^{2} \leq \alpha_{1}\left[d \left(h x_{2 n}\right.\right.} \\
& \left.\left.\quad f x_{2 n}\right) d\left(h x_{2 n+1}, g x_{2 n+1}\right)\right] \\
& +\alpha_{2}\left[d \left(h x_{2 n},\right.\right. \\
& \left.\left.\quad g x_{2 n+1}\right) d\left(h x_{2 n+1}, g x_{2 n+1}\right)\left(1+d\left(f x_{2 n}, h x_{2 n+1}\right)\right)\right] \\
& +\alpha_{3}\left[d \left(h x_{2 n},\right.\right. \\
& \left.\left.\quad h x_{2 n+1}\right) d\left(h x_{2 n+1}, g x_{2 n+1}\right)\left(1+d\left(f x_{2 n}, h x_{2 n+1}\right)\right)\right] \\
& +\alpha_{4}\left[d\left(h x_{2 n}, g x_{2 n+1}\right) d\left(h x_{2 n+1}, g x_{2 n+1}\right)\right] \\
& +\alpha_{5}\left[\left\{d\left(\left(h x_{2 n+1}, f x_{2 n}\right)\right)\right\}^{2}\right. \\
& \left.+\left\{d\left(h x_{2 n+1}, g x_{2 n+1}\right)\right\}^{2}\right] \leq \alpha_{1}\left[d \left(y_{2 n-1}\right.\right. \\
& \left.\left.\quad y_{2 n}\right) d\left(y_{2 n}, y_{2 n+1}\right)\right]+\alpha_{2}\left[d \left(y_{2 n-1},\right.\right. \\
& \left.\left.\quad y_{2 n+1}\right) d\left(y_{2 n}, y_{2 n+1}\right)\left(1+d\left(y_{2 n}, y_{2 n}\right)\right)\right] \\
& +\alpha_{3}\left[d \left(y_{2 n-1},\right.\right. \\
& \left.\left.\quad y_{2 n}\right) d\left(y_{2 n}, y_{2 n+1}\right)\left(1+d\left(y_{2 n}, y_{2 n}\right)\right)\right] \\
& +\alpha_{4}\left[d\left(y_{2 n-1}, y_{2 n+1}\right) d\left(y_{2 n+1}, y_{2 n}\right)\right] \\
& \left.\quad\left\{d\left(y_{2 n}, y_{2 n}\right)\right\}^{2}+\left\{d\left(y_{2 n}, y_{2 n+1}\right)\right\}^{2}\right]
\end{aligned}
$$

Or

$$
\begin{aligned}
d\left(y_{2 n}, y_{2 n+1}\right) & \\
\leq & \alpha_{1} d\left(y_{2 n-1}, y_{2 n}\right)+\alpha_{2} d\left(y_{2 n-1}, y_{2 n+1}\right) \\
& +\alpha_{3} d\left(y_{2 n-1}, y_{2 n}\right)+\alpha_{4} d\left(y_{2 n-1}, y_{2 n+1}\right) \\
& +\alpha_{5} d\left(y_{2 n}, y_{2 n+1}\right) \\
\leq & \alpha_{1} d\left(y_{2 n-1}, y_{2 n}\right) \\
& +s \alpha_{2}\left[d\left(y_{2 n-1}, y_{2 n}\right)+d\left(y_{2 n}, y_{2 n+1}\right)\right] \\
& +\alpha_{3} d\left(y_{2 n-1}, y_{2 n}\right) \\
& +s \alpha_{4}\left[d\left(y_{2 n-1}, y_{2 n}\right)+d\left(y_{2 n}, y_{2 n+1}\right)\right] \\
& +\alpha_{5} d\left(y_{2 n}, y_{2 n+1}\right) \\
\leq & \left(s \alpha_{2}+s \alpha_{4}+\alpha_{5}\right) d\left(y_{2 n}, y_{2 n+1}\right) \\
& +\left(\alpha_{1}+s \alpha_{2}+\alpha_{3}+s \alpha_{4}\right) d\left(y_{2 n}, y_{2 n-1}\right) \\
{[1-} & \left.\left(s \alpha_{2}+s \alpha_{4}+\alpha_{5}\right)\right] d\left(y_{2 n}, y_{2 n+1}\right)
\end{aligned}
$$

$$
\begin{gathered}
\leq\left(\alpha_{1}+s \alpha_{2}+\alpha_{3}+s \alpha_{4}\right) d\left(y_{2 n}, y_{2 n-1}\right) \Longrightarrow \\
d\left(y_{2 n+1}, y_{2 n}\right) \leq \frac{\left(\alpha_{1}+s \alpha_{2}+\alpha_{3}+s \alpha_{4}\right)}{\left[1-\left(s \alpha_{2}+s \alpha_{4}+\alpha_{5}\right)\right]} d\left(y_{2 n}, y_{2 n-1}\right) \Longrightarrow \\
d\left(y_{2 n+1}, y_{2 n}\right) \leq \lambda d\left(y_{2 n}, y_{2 n-1}\right),
\end{gathered}
$$

where

$$
\lambda=\frac{\left(\alpha_{1}+s \alpha_{2}+\alpha_{3}+s \alpha_{4}\right)}{\left[1-\left(s \alpha_{2}+s \alpha_{4}+\alpha_{5}\right)\right]}<\frac{1}{s} .
$$

Similarly, it can be shown that

$$
d\left(y_{2 n+1}, y_{2 n+2}\right) \leq \lambda d\left(y_{2 n}, y_{2 n+1}\right) \text {. }
$$

Therefore, for all $n \in N$ we can get

$$
d\left(y_{n+1}, y_{n+2}\right) \leq \lambda d\left(y_{n}, y_{n+1}\right) \leq \cdots \leq \lambda^{n+1} d\left(y_{0}, y_{1}\right) \text {. }
$$

Now, for any $m>n$, we have

$$
\begin{aligned}
d\left(y_{n}, y_{m}\right) \leq & d\left(y_{n}, y_{n+1}\right)+d\left(y_{n+1}, y_{n+2}\right)+\cdots \\
& +d\left(y_{m-1}, y_{m}\right) \\
\leq & {\left[\lambda^{n}+\lambda^{n+1}+\cdots+\lambda^{m-1}\right] d\left(y_{1}, y_{0}\right) } \\
\leq & \frac{\lambda^{n}}{(1-\lambda)} d\left(y_{1}, y_{0}\right) .
\end{aligned}
$$

Therefore, from Lemma 13, we have

$$
d\left(y_{n}, y_{m}\right) \leq \frac{s \lambda^{n}}{(1-s \lambda)} d\left(y_{1}, y_{0}\right) \longrightarrow 0
$$

as $m, n \longrightarrow \infty$,

where $s \lambda<1$. It follows that the sequence $\left\{y_{n}\right\}$ is a Cauchy sequence and, by the completeness of $X,\left\{y_{n}\right\}$ converges to some $y \in X$.

Therefore,

$$
\begin{aligned}
\lim _{n \rightarrow \infty} y_{n} & =\lim _{n \rightarrow \infty} f x_{2 n}=\lim _{n \rightarrow \infty} g x_{2 n+1}=\lim _{n \rightarrow \infty} h x_{2 n+1} \\
& =\lim _{n \rightarrow \infty} h x_{2 n+2}=y .
\end{aligned}
$$

Since $h(X)$ is a complete subspace of $X$, there exists $u, y \in X$ such that $h u=y$. If $g u \neq y$, using (34), we get

$$
\begin{aligned}
& {\left[d\left(f x_{2 n}, g u\right)\right]^{2}} \\
& \leq \alpha_{1}\left[d\left(h x_{2 n}, f x_{2 n}\right) d(h u, g u)\right] \\
& \quad+\alpha_{2}\left[d\left(h x_{2 n}, g u\right) d(h u, g u)\left(1+d\left(f x_{2 n}, h u\right)\right)\right] \\
& \quad+\alpha_{3}\left[d\left(h x_{2 n}, h u\right) d(h u, g u)\left(1+d\left(f x_{2 n}, h u\right)\right)\right] \\
& \quad+\alpha_{4}\left[d\left(h x_{2 n}, g u\right) d(h u, g u)\right] \\
& \quad+\alpha_{5}\left[\left\{d\left(h u, f x_{2 n}\right)\right\}^{2}+\{d(h u, g u)\}^{2}\right]
\end{aligned}
$$


and, on taking limit as $n \rightarrow \infty$,

$$
\begin{aligned}
& {[d(y, g u)]^{2}} \\
& \leq \alpha_{1}[d(y, y) d(y, g u)] \\
& \quad+\alpha_{2}[d(y, g u) d(y, g u)(1+d(y, y))] \\
& \quad+\alpha_{3}[d(y, y) d(y, g u)(1+d(y, y))] \\
& \quad+\alpha_{4}[d(y, g u) d(y, g u)] \\
& \quad+\alpha_{5}\left[\{d(y, y)\}^{2}+\{d(y, g u)\}^{2}\right] \\
& \leq\left(\alpha_{2}+\alpha_{4}+\alpha_{5}\right)[d(y, g u)]^{2} \Longrightarrow \\
& {\left[1-\left(\alpha_{2}+\alpha_{4}+\alpha_{5}\right)\right][d(y, g u)]^{2} \leq 0,}
\end{aligned}
$$

which is possible only if $[d(y, g u)]^{2}=0 \Rightarrow y=g u$. It follows that $h u=y=g u$. Since the pair of mappings $\{g, h\} g$ is weakly compatible, we have $g h u=h g u$ and so

$$
g y=h y
$$

If $y \neq g y$, by (34), we get the following.

$$
\begin{aligned}
& \text { As } n \rightarrow \infty \text {, we have } \\
& \lim _{n \rightarrow \infty}\left[d\left(f x_{2 n}, g y\right)\right]^{2} \\
& \leq \lim _{n \rightarrow \infty} \alpha_{1}\left[d_{1}\left(h x_{2 n}, f x_{2 n}\right) d(h y, g y)\right] \\
& +\alpha_{2}\left[d\left(h x_{2 n}, g y\right) d(h y, g y)\left(1+d\left(f x_{2 n}, h y\right)\right)\right] \\
& +\alpha_{3}\left[d\left(h x_{2 n}, h y\right) d(h y, g y)(1+d(y, y))\right] \\
& +\alpha_{4}\left[d\left(h x_{2 n}, g y\right) d(h y, g y)\right] \\
& +\alpha_{5}\left[\left\{d\left(h y, f x_{2 n}\right)\right\}^{2}+\{d(h y, g y)\}^{2}\right] \\
& \leq \alpha_{1}[d(y, y) d(g y, g y)] \\
& +\alpha_{2}[d(y, g y) d(y, g y)(1+d(y, y))] \\
& +\alpha_{3}[d(y, y) d(y, g y)(1+d(y, y))] \\
& +\alpha_{4}[d(y, g y) d(y, g y)] \\
& +\alpha_{5}\left[\{d(y, y)\}^{2}+\{d(y, g y)\}^{2}\right] \\
& \leq\left(\alpha_{2}+\alpha_{4}+\alpha_{5}\right)[d(y, g)]^{2} \Longrightarrow \\
& {\left[1-\left(\alpha_{2}+\alpha_{4}+\alpha_{5}\right)\right][d(y, g y)]^{2},}
\end{aligned}
$$

and the above inequality is possible only if $[d(y, g y)]^{2}=0 \Rightarrow$ $y=g y$. Therefore, by (46) we get

$$
y=g y=h y .
$$

Again, if $y \neq f u$, by (34) we have the following.
Taking limit as $n \rightarrow \infty$, we have

$$
\begin{aligned}
& {\left[d\left(f u, g x_{2 n+1}\right)\right]^{2}=[d(y, f u)]^{2} \leq \alpha_{1}[d(h u,} \\
& \left.\quad f u) d\left(h x_{2 n+1}, g x_{2 n+1}\right)\right] \\
& \quad+\alpha_{2}[d(h u, \\
& \left.\left.\quad g x_{2 n+1}\right) d\left(h x_{2 n+1}, g x_{2 n+1}\right)\left(1+d\left(f u, h x_{2 n+1}\right)\right)\right] \\
& \quad+\alpha_{3}[d(h u, \\
& \left.\left.\quad h x_{2 n+1}\right) d\left(h x_{2 n+1}, g x_{2 n+1}\right)\left(1+d\left(f u, h x_{2 n+1}\right)\right)\right] \\
& \quad+\alpha_{4}\left[d\left(h u, g x_{2 n+1}\right) d\left(h x_{2 n+1}, g x_{2 n+1}\right)\right] \\
& \quad+\alpha_{5}\left[\left\{d\left(h x_{2 n+1}, f u\right)\right\}^{2}+\left\{d\left(h x_{2 n+1}, g x_{2 n+1}\right)\right\}^{2}\right] \\
& \quad \leq \alpha_{1}[d(y, f u) d(y, y)]+\alpha_{2}[d(y, \\
& \quad y) d(y, y)(1+d(f u, y))] \\
& \quad+\alpha_{3}[d(y, y) d(y, y)(1+d(f u, y))]+\alpha_{4}[d(y, \\
& \quad y) d(y, y)]+\alpha_{5}\left[\{d(y, f u)\}^{2}\right. \\
& \left.+\{d(y, y)\}^{2}\right] \leq \alpha_{5}[d(y, f u)]^{2} \Longrightarrow \\
& \left(1-\alpha_{5}\right)[d(y, f u)]^{2} \leq 0,
\end{aligned}
$$

and this is possible only if $[d(f u, y)]^{2}=0 \Rightarrow y=f u$. Since $f$ and $h$ are weakly compatible, $f h u=h f u$ :

$$
\Longrightarrow f y=g y \text {. }
$$

By (48) and (50) we have

$$
f y=g y=h y=y .
$$

Thus $y$ is the unique common fixed point of $f, g$, and $h$.

Uniqueness. In order to see the uniqueness of the common fixed point, let $y_{1}$ and $y_{2}$ be two distinct common fixed points of $f, g$, and $h$ such that $y_{1} \neq y_{2}$. Then by using (34) we get

$$
\begin{aligned}
& {\left[d\left(y_{1}, y_{2}\right)\right]^{2}=\left[d\left(f y_{1}, g y_{2}\right)\right]^{2}} \\
& \quad \leq \alpha_{1}\left[d\left(h y_{1}, f y_{1}\right) d\left(h y_{2}, g y_{2}\right)\right] \\
& \quad+\alpha_{2}\left[d\left(h y_{1}, g y_{2}\right) d\left(h y_{2}, g y_{2}\right)\left(1+d\left(f y_{1}, h y_{2}\right)\right)\right] \\
& \quad+\alpha_{3}\left[d\left(h y_{1}, h y_{2}\right) d\left(h y_{2}, g y_{2}\right)\left(1+d\left(f y_{1}, h y_{2}\right)\right)\right] \\
& \quad+\alpha_{4}\left[d\left(h y_{1}, g y_{2}\right) d\left(h y_{2}, g y_{2}\right)\right] \\
& \quad+\alpha_{5}\left[\left\{d\left(h y_{2}, f y_{1}\right)\right\}^{2}+\left\{d\left(h y_{2}, g y_{2}\right)\right\}^{2}\right] \\
& \quad \leq \alpha_{1}\left[d\left(y_{1}, y_{1}\right) d\left(y_{2}, y_{2}\right)\right] \\
& \quad+\alpha_{2}\left[d\left(y_{1}, y_{2}\right) d\left(y_{2}, y_{2}\right)\left(1+d\left(y_{1}, y_{2}\right)\right)\right] \\
& \quad+\alpha_{3}\left[d\left(y_{1}, y_{2}\right) d\left(y_{2}, y_{2}\right)\left(1+d\left(y_{1}, y_{2}\right)\right)\right]
\end{aligned}
$$




$$
\begin{aligned}
& \quad+\alpha_{4}\left[d\left(y_{1}, y_{2}\right) d\left(y_{2}, y_{2}\right)\right] \\
& \quad+\alpha_{5}\left[\left\{d\left(y_{2}, y_{1}\right)\right\}^{2}+\left\{d\left(y_{2}, y_{2}\right)\right\}^{2}\right] \\
& \quad \leq \alpha_{5}\left[d\left(y_{1}, y_{2}\right)\right]^{2} \Longrightarrow \\
& \left(1-\alpha_{5}\right)\left[d\left(y_{1}, y_{2}\right)\right]^{2} \leq 0,
\end{aligned}
$$

and the inequality is possible only if $\left[d\left(y_{1}, y_{2}\right)\right]^{2}=0$ which implies that $y_{1}=y_{2}$ and the common fixed point is unique.

Theorem 16 yields the following corollaries.

Corollary 17. Let $(X, d)$ be a complete b-metric space with coefficient $s \geq 1$ and $f, g, h$ be a self-mapping of $X$ into itself satisfying

$$
\begin{aligned}
& {[d(f x, g y)]^{2} \leq \alpha_{1}[d(h x, f x) d(h y, g y)} \\
& \quad+d(h x, g y) d(h y, g y)(1+d(f x, h y))] \\
& \quad+\alpha_{3}[d(h x, h y) d(h y, g y)(1+d(f x, h y)) \\
& \quad+d(h x, g y) d(h y, g y)],
\end{aligned}
$$

for all $x, y \in X$; and $\alpha_{1}, \alpha_{3} \geq 0$ such that $\alpha_{1}+\left(s^{2}+1\right) \alpha_{3}<1$ then $f, g$, and h have a unique common fixed point in $X$.

Proof. Putting $\alpha_{5}=0$ and $\alpha_{2}=\alpha_{1}$, and $\alpha_{4}=\alpha_{3}$ in Theorem 16, we get the required result.

Corollary 18. Let $(X, d)$ be a complete $b$-metric space and $f, g, h: X \rightarrow X$ be self-maps of $X$ into itself satisfying the inequality

$$
\begin{aligned}
& {[d(f x, g y)]^{2}} \\
& \quad \leq \alpha_{1}[d(h x, f x) d(h y, g y)] \\
& \quad+\alpha_{2}[d(h x, g y) d(h y, g y)(1+d(f x, h y))],
\end{aligned}
$$

for all $x, y \in X$, such that $\alpha_{1}+\left(s^{2}+s\right) \alpha_{2}<1$, and then $f, g, h$ have a unique common fixed point in $X$.

Proof. Putting $\alpha_{3}=\alpha_{4}=\alpha_{5}=0$ in Theorem 16 we get the required result.

Remark 19. Corollary 17, is the result of Pachpatte [12].

Remark 20. Corollary 18, is the result of Fisher [11].

Example 21. Let $X=\{3,4,5\}$, and $d: X \times X \rightarrow R^{+}$is defined as follows:

$$
\begin{aligned}
& d(3,4)=d(4,3)=1, \\
& d(5,4)=d(4,5)=\frac{20}{25}, \\
& d(3,5)=d(5,3)=\frac{1}{25}, \\
& d(3,3)=d(4,4)=d(5,5)=0 .
\end{aligned}
$$

Then, it is easy to verify that $(X, d)$ is a $b$-metric space with parameter $s=4 / 5$. Define the mappings $f, g, h: X \rightarrow X$ by

$$
\begin{aligned}
& f_{3}=f_{5}=4, \\
& f_{4}=5, \\
& g_{3}=g_{4}=g_{5}=5, \\
& h_{3}=4, \\
& h_{4}=3, \\
& h_{5}=5 .
\end{aligned}
$$

Then, for $\alpha_{1}=\alpha_{2}=\alpha_{3}=\alpha_{4}=\alpha_{5}=1 / 2$, the self-maps $f, g$, and $h$ satisfy all the conditions of Theorems 14 and 16 with 5 as the only common fixed point in $X$.

\section{Conflicts of Interest}

The authors declare that there are no conflicts of interest regarding the publication of this paper.

\section{References}

[1] S. Banach, "Sur les opérations dans les ensembles abstraits et leur application aux équations intégrales," Fundamenta Mathematicae, vol. 3, pp. 133-181, 1922.

[2] I. A. Bakhtin, "The contraction mapping principle in almost metric space," Functional Analysis and its Applications, vol. 30, pp. 26-37, 1989.

[3] S. Czerwik, "Contraction mapping in b-metric spaces," Acta Mathematica et Informatica Universitatis Ostraviensis, vol. 1, no. 1, pp. 5-11, 1993.

[4] S. Czerwik, "Nonlinear set-valued contraction mappings in b-metric spaces," Atti del Seminario Matematico e Fisico dell'Università di Modena, vol. 46, no. 2, pp. 263-276, 1998.

[5] M. Boriceanu, "Strict fixed point theorems for multivalued operators in b-metric spaces," International Journal of Modern Mathematics, vol. 4, no. 3, pp. 285-301, 2009.

[6] M. Bota, A. Molnar, and C. Varga, "On Ekeland's variational principle in b-metric spaces," Fixed Point Theory and Applications, vol. 12, no. 1, pp. 21-28, 2011.

[7] C. Chen, L. Wen, J. Dong, and Y. Gu, "Fixed point theorems for generalized F-contraction in b-metric like spaces," Journal of Nonlinear Sciences and Applications, vol. 9, no. 5, pp. 2161-2174, 2016.

[8] N. Hussain and M. H. Shah, "KKM mapping in cone b-metric spaces," Computers \& Mathematics with Applications, vol. 62, no. 4, pp. 1677-1684, 2011.

[9] M. A. Kutbi, E. Karapinar, J. Ahmad, and A. Azam, "Some fixed point results for multi-valued mappings in b-metric spaces," Journal of Inequalities and Applications, vol. 2014, article 126, 2014.

[10] S. Shukla, "Partial b-metric spaces and fixed point theorems," Mediterranean Journal of Mathematics, vol. 11, no. 2, pp. 703711, 2014

[11] B. Fisher, "Fixed point and constant mappings on metric spaces," Atti della Accademia Nazionale dei Lincei. Rendiconti. Classe di Scienze Fisiche, Matematiche e Naturali, vol. 61, no. 5, pp. 329-332 (1977), 1976. 
[12] B. G. Pachpatte, "On certain fixed point mappings in metric spaces," Journal of the Maulana Azad College of Technology, vol. 13, pp. 59-63, 1980.

[13] P. L. Sharma and M. K. Sahu, "A unique fixed point theorem in complete metric space," Acta Ciencia Indica. Mathematics, vol. 17, no. 4, pp. 685-688, 1991.

[14] M. Arshad, A. Azam, and P. Vetro, "Some common fixed point results in cone metric spaces," Fixed Point Theory and Applications, vol. 2009, Article ID 493965, 2009.

[15] M. Abbas and G. Jungck, "Common fixed point results for noncommuting mappings without continuity in cone metric spaces," Journal of Mathematical Analysis and Applications, vol. 341, no. 1, pp. 416-420, 2008.

[16] G. Jungck, "Common fixed points for noncontinuous nonself maps on nonmetric spaces," Far East Journal of Mathematical Sciences, vol. 4, no. 2, pp. 199-215, 1996. 


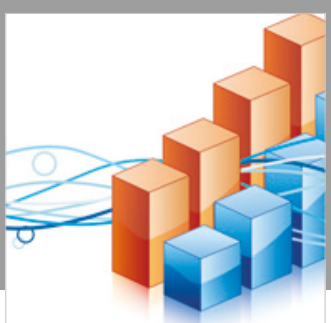

Advances in

Operations Research

\section{-n-m}
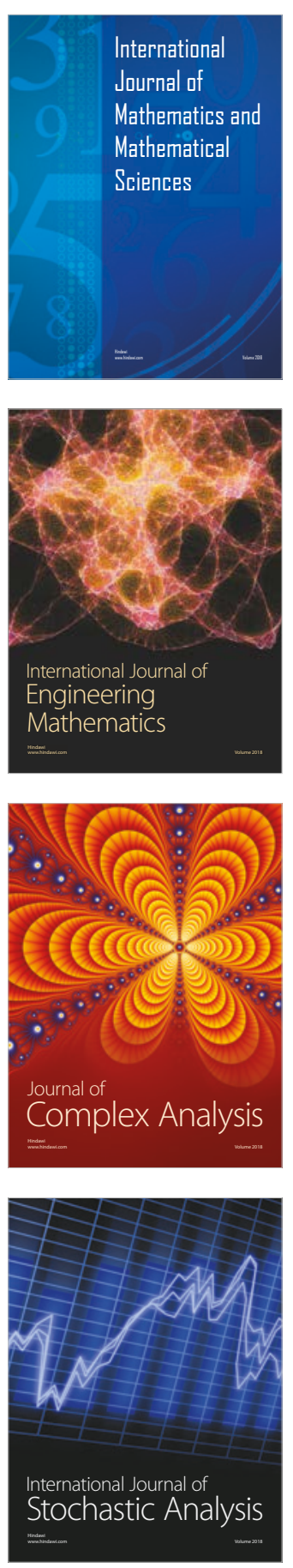
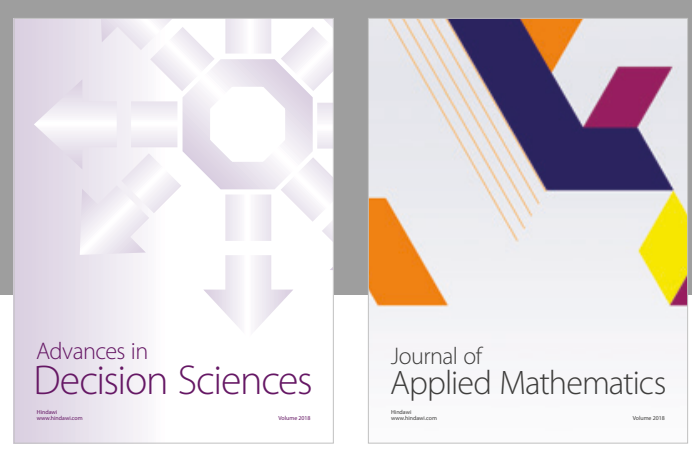

Journal of

Applied Mathematics
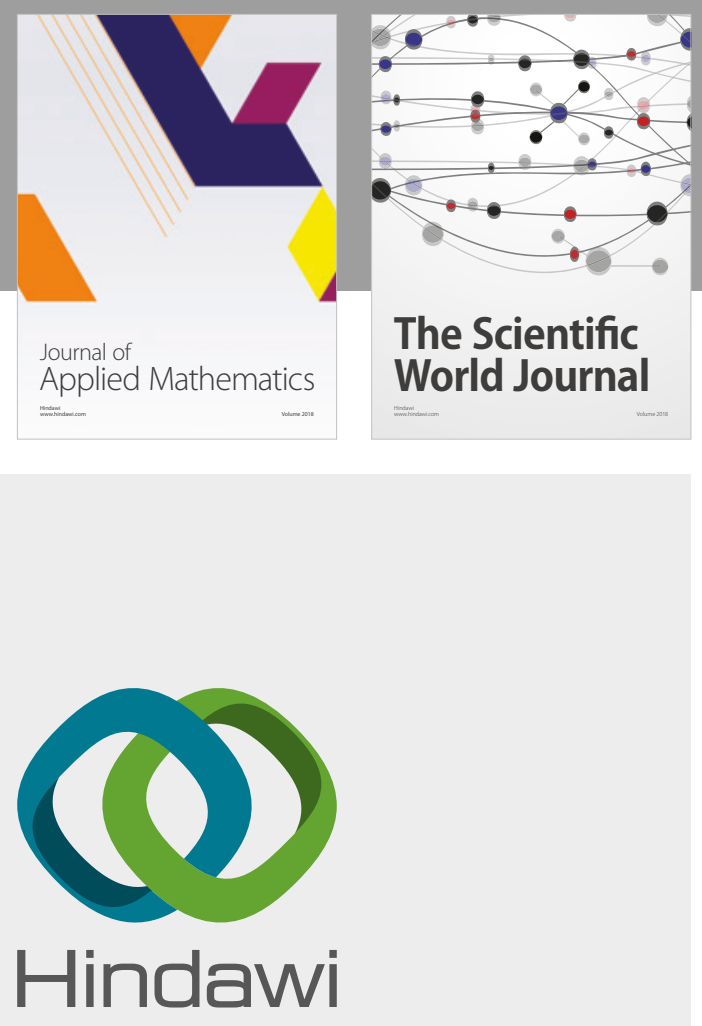

Submit your manuscripts at

www.hindawi.com

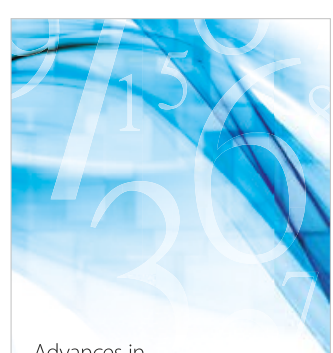

Advances in
Numerical Analysis
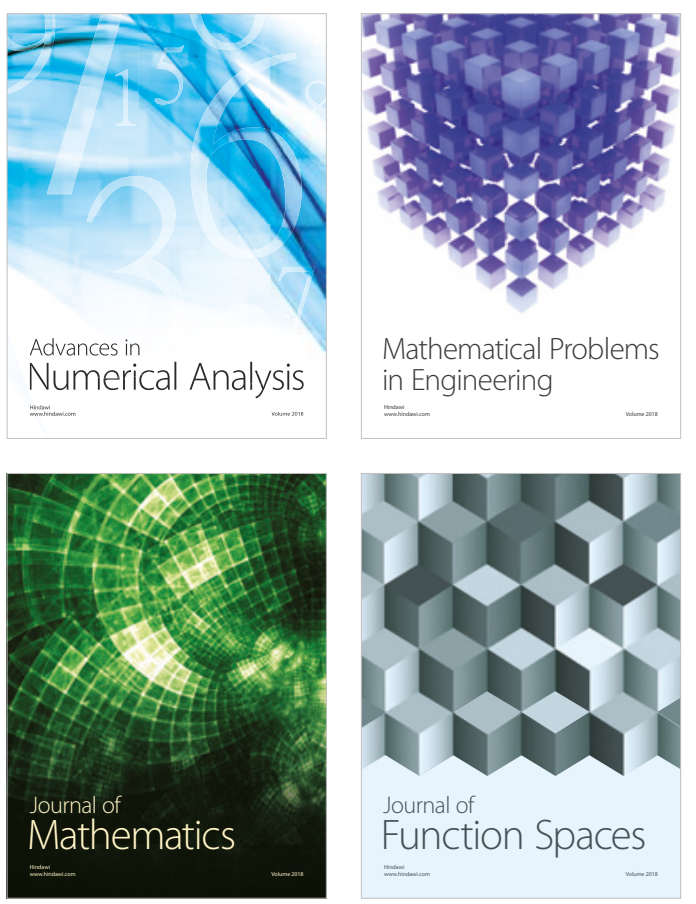

Mathematical Problems in Engineering

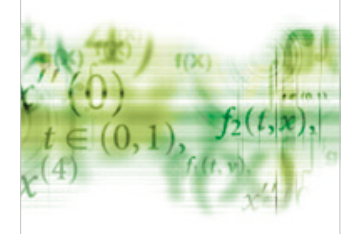

International Journal of

Differential Equations

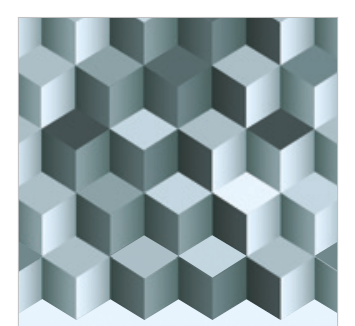

Journal of

Function Spaces

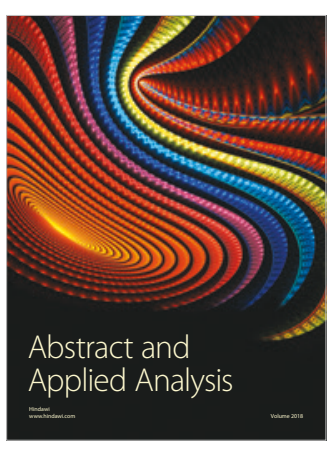

The Scientific

World Journal

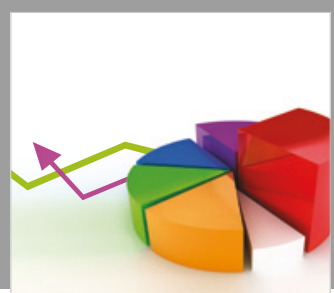

Journal of

Probability and Statistics
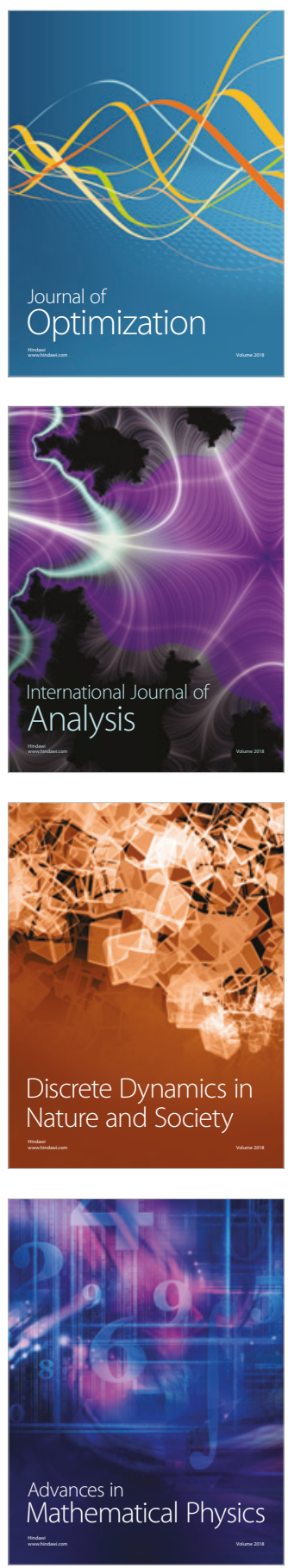